\title{
DIVERSITY OF ANGIOSPERM FLORA OF KUAKATA NATIONAL PARK, PATUAKHALI DISTRICT, BANGLADESH
}

\author{
M. AZIZAR RAHAMAN ${ }^{1}$, MD. AZIZUR RAHMAN ${ }^{2}$ AND \\ MOHAMMAD ZASHIM UDDIN ${ }^{3}$ \\ ${ }^{1}$ Wildlife Center, Ban Bhaban, Mohakhali, Dhaka-1212, Bangladesh \\ ${ }^{2}$ Department of Botany, Chittagong University, Bangladesh \\ ${ }^{3}$ Department of Botany, Dhaka University, Bangladesh
}

\begin{abstract}
The article mainly highlights the angiosperm diversity of Kuakata National Park (KNP) of Patuakhali district. Angiosperm diversity assessment in the park was conducted in between 2015 and 2016. A total of 265 plant species belonging to 75 families and 204 genera was identified from this National Park. Tree species of the park are represented by 89 , shrubs by 45 , herbs by 94 , climbers by 31 and epiphytes by 6 species. In Magnoliopsida (dicots), Fabaceae is the largest family represented by 14 species, whereas in Liliopsida (monocots), Poaceae is the largest family represented by 13 species. The plant species recorded from the National Park were distributed in different habitats and maximum species were recorded in plantation areas(108) followed by homesteads (61), cultivated land(38), roadsides (35) and mangrove areas (23). The study has reported the presence of medicinal plants, wildlife supporting plants, exotics and invasive plants and rare plants in park. The presence of edible species in the National Park is very rare. The introduction of exotics species into the National Park has been accepted. As the presence of exotics in park, local flora will be faced great challenges in future for their existence. This article also focused conservation values, management concerns and some actions for conservation of angiosperm diversity in the National Park. The present angiosperm diversity assessment in the park is very preliminary and based on this sound conclusion cannot be made yet.
\end{abstract}

Key words: Diversity, Angiosperm flora, Kuakata national park, Patuakhali district, Bangladesh

\section{Introduction}

Kuakata National Park (KNP) is the $12^{\text {th }}$ declared National Park of Bangladesh and a part of the Reserved Forest of Patuakhali Coastal Forest Division. Initially it was declared as an Eco-Park in 2005. Later Kuakata Eco-Park was gazetted as a National Park in 2010 (Gazette notification of Ministry of Environment and Forests no. MoEF/Forest Section2/02/National Park/10/2010/509, Dated: $24 / 10 / 2010$ as per power given under the provisions of Article 23 (3) of the Bangladesh Wildlife (Preservation) (Amendment) Act 1974). It is situated in the southern part of Bangladesh under Kalapara Upazila of Patuakhali district and geographical location is in between including $21^{\circ} 49^{\prime} 16^{\prime \prime} \mathrm{N}$ and $90^{\circ} 07^{\prime} 11^{\prime \prime} \mathrm{E}$. The park is bordered by the Bay of Bengal to the east, south and west

\footnotetext{
${ }^{1}$ Corresponding author : E-mail:alam027456@gmail.com
} 
including the Andharmanik River and WAPDA embankment to the north. The National Park has an area of 1613 ha with Gangamati, Khajura and Kuakata forest beats on the seashore, comprises coastal mangrove plantations (BFD 2012).

Kuakata National Park enjoys tropical maritime climate characterized by high rain during monsoon. The average temperature of KNP ranges between $21.71^{\circ} \mathrm{C}$ to $29.41^{\circ} \mathrm{C}$ and average annual rainfall is about $2657 \mathrm{~mm} /$ year. The soil of the area consists of calcareous alluvium, acid phosphate soil, grey floodplain and grey piedmont soils. These soils are saline and the $\mathrm{P}^{\mathrm{H}}$ values are neutral to slightly alkaline. KNP frequently was affected several serious cyclones during last couples of years. The park area is experiencing rapid erosion mainly at the south and west parts and is more threatened due to regular sea wave actions (BFD 2006).

The diversity of plants is very much essential in shaping of human civilization in recent days. Unfortunately, such diversity has been eroding in alarming rate from the nature before evaluation and documentation. At the end of 19 th century the head of states from all over the world had realized this burning issue. In 1992 world leaders in Earth Summit in Rieo De Janerio formulated biodiversity conservation policy including agenda 21 which had also given importance on the documentation and sustainable utilization of traditional knowledge of plant diversity. After the convention the assessment works of plant diversity in different countries of the world is in progress (Uddin and Abiabdullah 2016). In case of Bangladesh angiosperm diversity assessment of different National Parks and Wildlife Sanctuaries had already been started (Khan et al. 1994, Rahman and Hassan 1995, Uddin et al. 1998, Uddin and Rahman 1999, Khan and Huq 2001, Uddin et al. Uddin and Hassan 2004, 2010; 2011, Uddin et al. 2013, 2015, Sajib et al. 2015 and Uddin and Abiabdullah 2016). Literature review revealed that so far no work in available on the angiosperm diversity of Kuakata National Park. For the management of the park, baseline data on the angiosperm diversity are essential. In the present study an attempt was taken to achieve the following objectives: (a) to document the angiosperms diversity, (b) to highlights management concerns of the park and (c) to recommend some conservation actions for Kuakata National Park.

\section{Materials and Methods}

Extensive floristic survey had been conducted in different seasons of the year of 2015 and 2016 (Hyland 1972, Balick et al. 1982 and Alexiades 1996). The study included plantation areas, mangrove areas, cultivated land, roadside and homestead areas. Particular efforts were given to find species of conservation concern including threatened and rare species. Sample size was determined using species area curve or species time curve following Goldsmith and Harrison (1976). Maximum identification of species was done in the field sites and rest of the specimens was collected and processed using standard herbarium techniques (Hyland 1972). Identification was done by consulting 
different Floras (Uddin and Hassan 2004, Siddiqui et al. 2007 and Ahmed et al. 2008a, 2008b, 2009a, 2009b, 2009c, 2009d and 2009e).

The updated nomenclatures of the species are integrated by following Siddiqui et al. ( 2007) and Ahmed et al. (2008a, 2008b, 2009a, 2009b, 2009c, 2009d and 2009e). Threatened categories of plants were confirmed with the help of Khan et al. (2001) and Ara et al. (2013). Some noxious exotic plant species were also identified comparing with the reports of Hossain and Pasha (2004) and Akter and Zuberi (2009). Families were arranged according to Cronquist (1981). Voucher specimens are preserved at Wildlife Center Herbarium (WCH), Bangladesh Forest Department.

\section{Results and Discussion}

A total of 265 plant species belonging to75 families and 204 genera was identified from the Kuakata National Park. For each plant species scientific name, local name, family, habit and habitat are presented in Table 1. Among the families, Fabaceae, Poaceae, Caesalpiniaceae, Euphorbiaceae, Malvaceae, Moraceae, Solanaceae, Convolvulaceae, Asteraceae, Apocynaceae, Mimosaceae, Verbenaceae, Amaranthaceae, Cyperaceae, Acanthanceae and Zingiberaceae were found to be most common. By analyzing habit diversity it was found trees by 89 , shrubs by 45 , herbs by 94 , climbers by 31 and epiphytes by 6 species. In Magnoliopsida (dicots), Fabaceae is the largest family represented by 14 species, whereas in Liliopsida (monocots), Poaceae is the largest family represented by 13 species. The plant species recorded from the National Park was scattered in different habitats. Among the habitats, maximum species were recorded in plantation areas (108) followed by homesteads (61), cultivated land (38), roadsides (35) and mangrove areas (23). Most of the plant species in the plantation areas, homesteads and roadsides were introduced by the forest department and local people. The number of edible plants was found minimum in the park.

During the study, much concentration was given in the following habitats: The mangrove plantations were developed all around the National Park. Each year the newly accreted lands facing to the sea were undertaken by the forest department for plantation programs. The top canopy in the mangrove was occupied by Sonneratia apetala, S. caselaoris, Avicennia officinalis, Excoecaria agallocha and Bruguiera gymnorrhiza. Besides few representations of Heritiera fomes and Ceriops decandra were also detected in the park. The forest ground was covered mainly by the seedlings of Ex. agallocha, S. apetala and A. officinalis. In the forest periphery, the bush forming dominant species were Acanthus ilicifolius, Nipa fruticans and Ex. agallocha. The ground near the intertidal zone was mainly dominated by Pandanus foetidus, Phragmites karka and Saccharum spontaneum. Most common climbers in the mangrove forest were Derris scandens, D. trifolia, Ipomoea pes-caprae, Ichnocarpus frutescens and Desmodium heterocarpon. Some members of sedge species including Cyperus diformis and C. kyllingia were observed in this zone. The banks of the tidal zone were dominated by a good number of tree species 
Table 1. Angiosperms diversity of Kuakata National Park.

\begin{tabular}{|c|c|c|c|c|}
\hline Scientific name & Family & Local name & Habit & Habitat \\
\hline $\begin{array}{l}\text { Ablemoschus esculentus (L.) } \\
\text { Moench }\end{array}$ & Malvaceae & Vendi & Herb & Homestead \\
\hline Abroma augusta (L.) L.f. & Sterculiaceae & Ulatkambal & Tree & $\begin{array}{l}\text { Plantation } \\
\text { areas }\end{array}$ \\
\hline Abrus precatorius $\mathrm{L}$. & Fabaceae & Ratti & Climber & $\begin{array}{l}\text { Plantation } \\
\text { areas }\end{array}$ \\
\hline Abutilon indicum (L.) Sweet & Malvaceae & Abtilun & Shrub & Roadside \\
\hline $\begin{array}{l}\text { Acacia auriculiformis A.Cunn. ex } \\
\text { Benth. \& Hook. }\end{array}$ & Mimosaceae & Akashmoni & Tree & $\begin{array}{l}\text { Plantation } \\
\text { areas }\end{array}$ \\
\hline Acacia farnesiana (L. f.)Willd. & Mimosaceae & Khaia babla & Tree & $\begin{array}{l}\text { Plantation } \\
\text { areas }\end{array}$ \\
\hline Acacia mangium Willd. & Mimosaceae & Belgium & Tree & $\begin{array}{l}\text { Plantation } \\
\text { areas }\end{array}$ \\
\hline Acacia nilotica $\mathrm{L}$. & Mimosaceae & Babla & Tree & $\begin{array}{l}\text { Plantation } \\
\text { areas }\end{array}$ \\
\hline Acanthus ilicifolius L. & Acanthaceae & Hergoza & Shrub & $\begin{array}{l}\text { Mangrove } \\
\text { areas }\end{array}$ \\
\hline Achyranthes aspera L. & Amaranthaceae & Apang & Herb & Homestead \\
\hline Adenanthera pavonina $\mathrm{L}$. & Mimosaceae & Raktakambal & Tree & Homestead \\
\hline Adhatoda zeylanica Mdikus & Acanthaceae & Bashak & Shrub & Homestead \\
\hline Aegle marmelos (L.) Corr. & Rutaceae & Bel & Tree & Homestead \\
\hline Aerides multiflora Roxb. & Orchidaceae & Aerid & Epiphyte & $\begin{array}{l}\text { Plantation } \\
\text { areas }\end{array}$ \\
\hline Aerides odorata Lour. & Orchidaceae & Aerid & Epiphyte & $\begin{array}{l}\text { Plantation } \\
\text { areas }\end{array}$ \\
\hline Ageratum conyzoides L. & Asteraceae & Fulkuri & Herb & Roadside \\
\hline Albizia lebbeck (L.)Benth. \& Hook. & Mimosaceae & Kalo koroi & Tree & Roadside \\
\hline Albizia lucidior (Steud.) Nielsen & Mimosaceae & Koroi & Tree & Homestead \\
\hline Albizia procera(Roxb.) Benth. & Mimosaceae & Sil-koroi & Tree & Roadside \\
\hline Allamanda cathartica $\mathrm{L}$. & Apocynaceae & Allamanda & Herb & $\begin{array}{l}\text { Plantation } \\
\text { areas }\end{array}$ \\
\hline Allophylus cobbe (L.) Raeuschel & Sapindaceae & Chita & Shrub & $\begin{array}{l}\text { Plantation } \\
\text { areas }\end{array}$ \\
\hline Alocasia macrorrhizos (L.) G. Don & Araceae & Mankachu & Herb & Homestead \\
\hline Alstonia scholaris L. & Apocynaceae & Chatim & Tree & $\begin{array}{l}\text { Plantation } \\
\text { areas }\end{array}$ \\
\hline $\begin{array}{l}\text { Alternanthera philoxeroides } \\
\text { (Mart.) } \\
\text { Griseb. }\end{array}$ & Amaranthaceae & Helencha & Herb & $\begin{array}{l}\text { Cultivated } \\
\text { land }\end{array}$ \\
\hline Amaranthus lividus L. & Amaranthaceae & Gobura notey & Herb & Roadside \\
\hline Amaranthus spinosus $\mathrm{L}$. & Amaranthaceae & Kanta-nutia & Herb & Roadside \\
\hline Amaranthus viridis $\mathrm{L}$. & Amaranthaceae & Notey sak & Herb & Homestead \\
\hline $\begin{array}{l}\text { Amorphophallus bulbifer (Roxb.) } \\
\text { Blume }\end{array}$ & Araceae & Oll & Herb & Homestead \\
\hline $\begin{array}{l}\text { Andrographis paniculata } \\
\text { (Burm.f.)Wall.ex Nees }\end{array}$ & Acanthaceae & Kalo megh & Herb & Homestead \\
\hline Annona reticulata $\mathrm{L}$. & Annonaceae & Ata & Tree & Homestead \\
\hline $\begin{array}{l}\text { Anthocephalus cadamba (Roxb.) } \\
\text { Miq. }\end{array}$ & Rubiaceae & Kadam & Tree & $\begin{array}{l}\text { Plantation } \\
\text { areas }\end{array}$ \\
\hline
\end{tabular}




\begin{tabular}{|c|c|c|c|c|}
\hline Scientific name & Family & Local name & Habit & Habitat \\
\hline $\begin{array}{l}\text { Aphanamixis polystachya (Wall.) } \\
\text { R. N. Parker }\end{array}$ & Meliaceae & Pitraj & Tree & $\begin{array}{l}\text { Plantation } \\
\text { areas }\end{array}$ \\
\hline Areca catechu $\mathrm{L}$ & Arecaceae & Supari & Tree & Homestead \\
\hline Artocarpus chaplasha Roxb. & Moraceae & Chapalish & Tree & $\begin{array}{l}\text { Plantation } \\
\text { areas }\end{array}$ \\
\hline Artocarpus heterophyllus Lamk. & Moraceae & Kanthal & Tree & Homestead \\
\hline Averrhoa bilimbi L. & Oxalidaceae & Bilimbi & Tree & Homestead \\
\hline Averrhoa carambola $\mathrm{L}$. & Oxalidaceae & Kamranga & Tree & Homestead \\
\hline Avicennia alba Blume & Verbenaceae & Sada baen & Tree & $\begin{array}{l}\text { Mangrove } \\
\text { areas }\end{array}$ \\
\hline Avicennia marina (Forssk.) Vierh. & Verbenaceae & Moricha baen & Tree & $\begin{array}{l}\text { Mangrove } \\
\text { areas }\end{array}$ \\
\hline Avicennia officinalis L. & Verbenaceae & Kala baen & Tree & $\begin{array}{l}\text { Mangrove } \\
\text { areas }\end{array}$ \\
\hline Azadirachta indica A. Juss. & Meliaceae & Neem & Tree & Homestead \\
\hline Bacopa monnieri (L.) Pennell & Scrophulariaceae & Brammi & Herb & $\begin{array}{l}\text { Cultivated } \\
\text { land }\end{array}$ \\
\hline Bambusa tulda Roxb. & Poaceae & Mitting bash & Tree & Homestead \\
\hline $\begin{array}{l}\text { Barringtonia acutangula (L.) } \\
\text { Gaertn. }\end{array}$ & Lecythidaceae & Hizol & Tree & $\begin{array}{l}\text { Mangrove } \\
\text { areas }\end{array}$ \\
\hline Bauhinia purpurea $\mathrm{L}$. & Caesalpiniaceae & Kanson & Tree & $\begin{array}{l}\text { Plantation } \\
\text { areas }\end{array}$ \\
\hline Blumea lacera (Burm. f.) DC. & Asteraceae & Kukurmuta & Herb & $\begin{array}{l}\text { Cultivated } \\
\text { land }\end{array}$ \\
\hline Blumea membranacea Wall. exDC. & Asteraceae & Shialutra & Herb & $\begin{array}{l}\text { Cultivated } \\
\text { land }\end{array}$ \\
\hline Bombax ceiba L. & Bombacaceae & Shimul & Tree & $\begin{array}{l}\text { Plantation } \\
\text { areas }\end{array}$ \\
\hline Borassus flabellifer L. & Arecaceae & Tal & Tree & Homestead \\
\hline Bridelia retusa (L.) A. Juss. & Euphorbiacea & Kata koi & Shrub & $\begin{array}{l}\text { Plantation } \\
\text { areas }\end{array}$ \\
\hline Bruguiera gymnorhiza (L.) Lamk. & Rhizophoraceae & Kakra & Tree & $\begin{array}{l}\text { Mangrove } \\
\text { areas }\end{array}$ \\
\hline Butea monosperma (Lamk.) Taub. & Fabaceae & Polash & Tree & $\begin{array}{l}\text { Plantation } \\
\text { areas }\end{array}$ \\
\hline $\begin{array}{l}\text { Caesalpinia pulcherrima (L.) } \\
\text { Swartz }\end{array}$ & Caesalpiniaceae & Radhachura & Tree & $\begin{array}{l}\text { Plantation } \\
\text { areas }\end{array}$ \\
\hline Cajanus cajan (L.) Millsp. & Fabaceae & Orhor & Shrub & Homestead \\
\hline Calamus erectus Roxb. & Arecaceae & Kadam Bet & Shrub & $\begin{array}{l}\text { Plantation } \\
\text { areas }\end{array}$ \\
\hline $\begin{array}{l}\text { Calotropis procera (Aiton) } \\
\text { Dryand }\end{array}$ & Asclepiadaceae & Akand & Shrub & Roadside \\
\hline Calophyllum innophyllum $\mathrm{L}$. & Clusiaceae & Punnal & Tree & $\begin{array}{l}\text { Plantation } \\
\text { areas }\end{array}$ \\
\hline $\begin{array}{l}\text { Calycopteris floribunda (Roxb.) } \\
\text { Lamk. }\end{array}$ & Combretaceae & Guicha lata & Climber & $\begin{array}{l}\text { Plantation } \\
\text { areas }\end{array}$ \\
\hline Carica papaya $\mathrm{L}$ & Caricaceae & Pepe & Shrub & Homestead \\
\hline Cassia alata $\mathrm{L}$. & Caesalpiniaceae & Dadmordan & Shrub & Roadside \\
\hline
\end{tabular}




\begin{tabular}{|c|c|c|c|c|}
\hline Scientific name & Family & Local name & Habit & Habitat \\
\hline Cassia fistula L. & Caesalpiniaceae & Sonalu & Tree & $\begin{array}{l}\text { Plantation } \\
\text { areas }\end{array}$ \\
\hline Cassia tora $\mathrm{L}$. & Caesalpiniaceae & Tora & Herb & Roadside \\
\hline Cassia siamea Lamk. & Caesalpiniaceae & Minjiri & Tree & $\begin{array}{l}\text { Plantation } \\
\text { areas }\end{array}$ \\
\hline Casuarina equisetifolia Forst. & Casuarinaceae & Jhau & Tree & $\begin{array}{l}\text { Plantation } \\
\text { areas }\end{array}$ \\
\hline Celosia cristataL. & Amaranthaceae & Morogful & Herb & $\begin{array}{l}\text { Cultivated } \\
\text { land }\end{array}$ \\
\hline Centella asiatica (L.) Urban & Apiaceae & Thankoni & Herb & $\begin{array}{l}\text { Cultivated } \\
\text { land }\end{array}$ \\
\hline $\begin{array}{l}\text { Ceriops decandra (Griff.) Ding. } \\
\text { Hou }\end{array}$ & Rhizophoraceae & Goran & Tree & $\begin{array}{l}\text { Mangrove } \\
\text { areas }\end{array}$ \\
\hline $\begin{array}{l}\text { Chrysalidocarpus lutescens (Bory) } \\
\text { H. Wendl. }\end{array}$ & Arecaceae & Areca palm & Shrub & $\begin{array}{l}\text { Plantation } \\
\text { areas }\end{array}$ \\
\hline $\begin{array}{l}\text { Chrysopogon aciculatus (Retz.) } \\
\text { Trin. }\end{array}$ & Poaceae & Premkanta & Herb & Roadside \\
\hline $\begin{array}{l}\text { Citrus aurantifolia } \\
\text { (Christm.\&Panzer) Swingle }\end{array}$ & Rutaceae & Labu & Shrub & Homestead \\
\hline Citrus maxima (Burm. F.) Merr. & Rutaceae & Jambura & Tree & Homestead \\
\hline Clerodendrum viscosumVent. & Verbenaceae & Bhant & Shrub & Roadside \\
\hline Clitoria ternatea $\mathrm{L}$. & Fabaceae & Aparjita & Climber & Homestead \\
\hline Coccinia grandis(L.) Voigt & Cucurbitaceae & Telakucha & Climber & $\begin{array}{l}\text { Plantation } \\
\text { areas }\end{array}$ \\
\hline Cocos nucifera $\mathrm{L}$. & Arecaceae & Narikel & Tree & Homestead \\
\hline $\begin{array}{l}\text { Codiaeum variegatum }(\mathrm{L} .) \text { Rumph } \\
\text { ex A. Juss. }\end{array}$ & Euphorbiaceae & Patabahar & Shrub & $\begin{array}{l}\text { Plantation } \\
\text { areas }\end{array}$ \\
\hline Colocasia esculenta (L.) Schott & Araceae & Kachu & Herb & Homestead \\
\hline Commelina benghalensis $\mathrm{L}$. & Commelinaceae & Kanchira & Herb & $\begin{array}{l}\text { Cultivated } \\
\text { land }\end{array}$ \\
\hline Cosmos bipinnatus Cav. & Asteraceae & Cosmos & Herb & $\begin{array}{l}\text { Cultivated } \\
\text { land }\end{array}$ \\
\hline $\begin{array}{l}\text { Costus speciosus (Koenig ex Retz.) } \\
\text { Smith }\end{array}$ & Costaceae & Keumul & Herb & $\begin{array}{l}\text { Plantation } \\
\text { areas }\end{array}$ \\
\hline Crinum asiaticum $\mathrm{L}$. & Liliaceae & Crinum & Herb & $\begin{array}{l}\text { Mangrove } \\
\text { areas }\end{array}$ \\
\hline Crotalaria pallida Ait. & Fabaceae & Jhunjhuni & Herb & Roadside \\
\hline Crotalaria juncea $\mathrm{L}$. & Fabaceae & Jhunjhuni & Herb & Roadside \\
\hline Croton bonplandianum Baill. & Euphorbiaceae & Croton & Herb & Roadside \\
\hline Cucurbita maxima Duchesne & Cucurbitaceae & Misti kumra & Climber & Homestead \\
\hline Curculigo orchioides Gaertn. & Liliaceae & Talmuli & Herb & $\begin{array}{l}\text { Plantation } \\
\text { areas }\end{array}$ \\
\hline Curcuma amada Roxb. & Zingiberaceae & Amada & Herb & $\begin{array}{l}\text { Plantation } \\
\text { areas }\end{array}$ \\
\hline Curcuma domestica Valet. & Zingiberaceae & Holud & Herb & Homestead \\
\hline Curcuma latifolia Rosc. & Zingiberaceae & Sadi & Herb & Roadside \\
\hline
\end{tabular}




\begin{tabular}{|c|c|c|c|c|}
\hline Scientific name & Family & Local name & Habit & Habitat \\
\hline $\begin{array}{l}\text { Curcuma zedoaria (Christm.) } \\
\text { Rosc. }\end{array}$ & Zingiberaceae & Shoti & Herb & $\begin{array}{l}\text { Plantation } \\
\text { areas }\end{array}$ \\
\hline Cuscuta reflexa Roxb. & Cuscutaceae & Shorna lata & Climber & Roadside \\
\hline Cymbidium aloifolium (L.) Sw. & Orchidaceae & Churi & Epiphyte & $\begin{array}{l}\text { Plantation } \\
\text { areas }\end{array}$ \\
\hline Cynodon dactylon (L.) Pers. & Poaceae & Durba & Herb & Roadside \\
\hline Cyperus cyperoides (L.) O. Ktze. & Cyperaceae & Sedge & Herb & $\begin{array}{l}\text { Cultivated } \\
\text { land }\end{array}$ \\
\hline Cyperus iria $\mathrm{L}$. & Cyperaceae & Sedge & Herb & $\begin{array}{l}\text { Cultivated } \\
\text { land }\end{array}$ \\
\hline Cyperus kyllingia Endl. & Cyperaceae & Sedge & Herb & $\begin{array}{l}\text { Mangrove } \\
\text { areas }\end{array}$ \\
\hline Cyperus rotundus $\mathrm{L}$. & Cyperaceae & Mutha grass & Herb & $\begin{array}{l}\text { Cultivated } \\
\text { land }\end{array}$ \\
\hline Dalbergia sissoo Roxb & Fabaceae & Sissoo & Tree & Plantation \\
\hline Datura metel L. & Solanaceae & Dhatura & Shrub & $\begin{array}{l}\text { areas } \\
\text { Plantation } \\
\text { areas }\end{array}$ \\
\hline Delonix regia (Hook) Raf. & Caesalpiniaceae & Krishnachura & Tree & $\begin{array}{l}\text { Plantation } \\
\text { areas }\end{array}$ \\
\hline $\begin{array}{l}\text { Dendrobium aphyllum (Roxb.) } \\
\text { Fischer }\end{array}$ & Orchidaceae & Dendrobium & Epiphyte & $\begin{array}{l}\text { Plantation } \\
\text { areas }\end{array}$ \\
\hline Dendrobium nobile Lindl. & Orchidaceae & Dendrobium & Epiphyte & $\begin{array}{l}\text { Plantation } \\
\text { areas }\end{array}$ \\
\hline Derris scandens (Roxb.) Benth. & Fabaceae & Kalilata & Climber & $\begin{array}{l}\text { Mangrove } \\
\text { areas }\end{array}$ \\
\hline Derris trifoliata Lour. & Fabaceae & Mellata & Climber & $\begin{array}{l}\text { Mangrove } \\
\text { areas }\end{array}$ \\
\hline Desmodium heterocarpon (L.) DC. & Fabaceae & - & Climber & $\begin{array}{l}\text { Mangrove } \\
\text { areas }\end{array}$ \\
\hline Dillenia indica $\mathrm{L}$. & Dilleniaceae & Chalta & Tree & Homestead \\
\hline Dioscorea alata $\mathrm{L}$. & Dioscoreaceae & Jora alu & Climber & Homestead \\
\hline Dioscorea bulbifera L. & Dioscoreaceae & Bon alu & Climber & Homestead \\
\hline Dioscorea pentaphylla $\mathrm{L}$. & Dioscoreaceae & - & Climber & $\begin{array}{l}\text { Plantation } \\
\text { areas }\end{array}$ \\
\hline $\begin{array}{l}\text { Diospyros malabarica (Desr.) } \\
\text { Kostel. }\end{array}$ & Ebenaceae & Deshigab & Tree & Homestead \\
\hline Dracaena spicata Roxb. & Agavaceae & Dracaena & Shrub & $\begin{array}{l}\text { Plantation } \\
\text { areas }\end{array}$ \\
\hline Duranta erecta $\mathrm{L}$. & Verbenaceae & Katamehedi & Shrub & $\begin{array}{l}\text { Plantation } \\
\text { areas }\end{array}$ \\
\hline Echinochloa colona (L.) Link & Poaceae & Shama grass & Herb & $\begin{array}{l}\text { Cultivated } \\
\text { land }\end{array}$ \\
\hline Eclipta prostrata $(\mathrm{L}$.) L. & Asteraceae & Keshoraj & Herb & $\begin{array}{l}\text { Cultivated } \\
\text { land }\end{array}$ \\
\hline Eichhornia crassipes (Mart.) Solms & Pontederiaceae & Kachuripana & Herb & $\begin{array}{l}\text { Cultivated } \\
\text { land }\end{array}$ \\
\hline Elaeocarpus tectorius (Lour.) Poir. & Elaeocarpaceae & Jalpai & Tree & Homestead \\
\hline Enhydra variegata $\mathrm{L}$ & Asteraceae & Helencha & Herb & $\begin{array}{l}\text { Cultivated } \\
\text { land }\end{array}$ \\
\hline
\end{tabular}




\begin{tabular}{|c|c|c|c|c|}
\hline Scientific name & Family & Local name & Habit & Habitat \\
\hline Erythrina indica Lam. & Fabaceae & Mandar & Tree & $\begin{array}{l}\text { Plantation } \\
\text { areas }\end{array}$ \\
\hline Eucalyptus camaldulensis Dehnh. & Myrtaceae & Eucalyptus & Tree & $\begin{array}{l}\text { Plantation } \\
\text { areas }\end{array}$ \\
\hline Euphorbia hirta L. & Euphorbiaceae & Dudhia & Herb & $\begin{array}{l}\text { Cultivated } \\
\text { land }\end{array}$ \\
\hline Euphorbia thymifolia $\mathrm{L}$. & Euphorbiaceae & Dudhia & Herb & $\begin{array}{l}\text { Cultivated } \\
\text { land }\end{array}$ \\
\hline Excoecaria agallocha $\mathrm{L}$. & Euphorbiaceae & Gewa & Tree & $\begin{array}{l}\text { Mangrove } \\
\text { areas }\end{array}$ \\
\hline Ficus benghalensis L. & Moraceae & Bot & Tree & Roadside \\
\hline Ficus benjamina $\mathrm{L}$. & Moraceae & $\begin{array}{l}\text { Jir } \\
\text { Dumur }\end{array}$ & Tree & $\begin{array}{l}\text { Plantation } \\
\text { areas }\end{array}$ \\
\hline Ficus hispidaL.f. & Moraceae & Dewall & Shrub & $\begin{array}{l}\text { Plantation } \\
\text { areas }\end{array}$ \\
\hline Ficus pumila $\mathrm{L}$. & Moraceae & dumar & Climber & $\begin{array}{l}\text { Plantation } \\
\text { areas }\end{array}$ \\
\hline Ficus racemosa $\mathrm{L}$. & Moraceae & Jagya dumar & Tree & Homestead \\
\hline Ficus virens Ait. & Moraceae & Pakur & Tree & Roadside \\
\hline Fimbristylis dichotoma (L.) Vahl & Cyperaceae & - & Herb & $\begin{array}{l}\text { Cultivated } \\
\text { land }\end{array}$ \\
\hline $\begin{array}{l}\text { Fimbristylis quinopangularis } \\
\text { (Vahl) Kunth. }\end{array}$ & Cyperaceae & - & Herb & $\begin{array}{l}\text { Cultivated } \\
\text { land }\end{array}$ \\
\hline Garcinia cowa Roxb. Choisy. & Clusiaceae & Kao & Tree & Homestead \\
\hline Gloriosa superba $\mathrm{L}$. & Liliaceae & Ulatchandal & Climber & $\begin{array}{l}\text { Plantation } \\
\text { areas }\end{array}$ \\
\hline $\begin{array}{l}\text { Glycosmis pentaphylla (Retz.) } \\
\text { DC. }\end{array}$ & Rutaceae & Datmajan & Shrub & Roadside \\
\hline Gmelina arborea Roxb. & Varbenaceae & Gamari & Tree & $\begin{array}{l}\text { Plantation } \\
\text { areas }\end{array}$ \\
\hline $\begin{array}{l}\text { Gymnopetalum chinense } \\
\text { (Lour.)Merr. }\end{array}$ & Cucurbitaceae & - & Climber & Homestead \\
\hline Hedyotis scandens Roxb. & Rubiaceae & Bish lata & Climber & $\begin{array}{l}\text { Plantation } \\
\text { areas }\end{array}$ \\
\hline Heliotropium indicum $\mathrm{L}$. & Boraginaceae & Hatisur & Herb & $\begin{array}{l}\text { Cultivated } \\
\text { land }\end{array}$ \\
\hline $\begin{array}{l}\text { Hemidesmus indicus (L.) R. Br. ex } \\
\text { Schott. }\end{array}$ & Asclepiadacae & Anantamul & Climber & $\begin{array}{l}\text { Plantation } \\
\text { areas }\end{array}$ \\
\hline Heritiera fomes Buch.-Ham. & Sterculiaceae & Sundari & Tree & $\begin{array}{l}\text { Mangrove } \\
\text { areas }\end{array}$ \\
\hline Hibiscus rosa sinensis L. & Malvaceae & Joba & Shrub & $\begin{array}{l}\text { Plantation } \\
\text { areas }\end{array}$ \\
\hline Hibiscus tilliaceus $\mathrm{L}$. & Malvaceae & Bolla & Shrub & $\begin{array}{l}\text { Plantation } \\
\text { areas }\end{array}$ \\
\hline $\begin{array}{l}\text { Hodgsonia macrocarpa (Blume) } \\
\text { Cogn. }\end{array}$ & Cucurbitaceae & Makal & Climber & $\begin{array}{l}\text { Plantation } \\
\text { areas }\end{array}$ \\
\hline $\begin{array}{l}\text { Holarrhena antidysenterica (L.) } \\
\text { Wall. ex Decne. }\end{array}$ & Apocynaceae & Kurchi & Shrub & $\begin{array}{l}\text { Plantation } \\
\text { areas }\end{array}$ \\
\hline Hopea odorata Roxb. & Dipterocarpaceae & Telsur & Tree & Homestead \\
\hline
\end{tabular}




\begin{tabular}{|c|c|c|c|c|}
\hline Scientific name & Family & Local name & Habit & Habitat \\
\hline $\begin{array}{l}\text { Hoya prasitica (Roxb.) Wall. ex } \\
\text { Wight }\end{array}$ & Apocynaceae & Hoya & Climber & Roadside \\
\hline Hyptis suaveolens (L.) Poit. & Lamiaceae & Tokma & Herb & Roadside \\
\hline $\begin{array}{l}\text { Ichnocarpus frutescens (L.) } \\
\text { W.T.Alton }\end{array}$ & Apocynaceae & Shamalata & Climber & $\begin{array}{l}\text { Mangrove } \\
\text { areas }\end{array}$ \\
\hline Imperata cylindrica (L.) Raeusch. & Poaeae & Ulu & Herb & Roadside \\
\hline Ipomoea aquatica Forssk. & Convolvulaceae & Kalmi & Climber & Homestead \\
\hline Ipomoea batata (L.) Lamk. & Convolvulaceae & Misti alu & Climber & Homestead \\
\hline Ipomoea fistulosa Mart. ex Choisy & Convolvulaceae & Dholkalmi & Shrub & Roadside \\
\hline Ipomoea pes-caprae (L.) R. Br. & Convolvulaceae & Chagalkhuri & Climber & $\begin{array}{l}\text { Mangrove } \\
\text { areas }\end{array}$ \\
\hline $\begin{array}{l}\text { Ischaemum polytrias indica } \\
\text { (Houtt.) Veldkamp. }\end{array}$ & Poaceae & Toto grass & Herb & $\begin{array}{l}\text { Cultivated } \\
\text { land }\end{array}$ \\
\hline Ixora acuminata Roxb. & Rubiaceae & Rangan & Shrub & $\begin{array}{l}\text { Plantation } \\
\text { areas }\end{array}$ \\
\hline Ixora Javanica (Blum) DC. & Rubiaceae & Rangan & Shrub & $\begin{array}{l}\text { Plantation } \\
\text { areas }\end{array}$ \\
\hline Ixora pavetta Andr. & Rubiaceae & Swet rangan & Shrub & $\begin{array}{l}\text { Plantation } \\
\text { areas }\end{array}$ \\
\hline Jatropha gossypiifolia $\mathrm{L}$. & Euphorbiaceae & Lal bharenda & Shrub & Homestead \\
\hline Justicia simplex $\mathrm{D}$. Don. & Acanthaceae & Jogathmardan & Shrub & $\begin{array}{l}\text { Plantation } \\
\text { areas }\end{array}$ \\
\hline $\begin{array}{l}\text { Kyllinga nemoralis (J.R.Forst. \& G. } \\
\text { Forst.) Dandy ex Hutch\&Dalziel }\end{array}$ & Cyperaceae & Sedge & Herb & $\begin{array}{l}\text { Cultivated } \\
\text { land }\end{array}$ \\
\hline Lablab purpureus (L.) Sweet & Fabaceae & Seem & Climber & Homestead \\
\hline Lagerstroemia speciosa (L.) Pers. & Lythraceae & Jarul & Tree & $\begin{array}{l}\text { Plantation } \\
\text { areas }\end{array}$ \\
\hline $\begin{array}{l}\text { Lannea coromandelica (Houtt.) } \\
\text { Merr. }\end{array}$ & Anacardiaceae & Jiga & Tree & $\begin{array}{l}\text { Plantation } \\
\text { areas }\end{array}$ \\
\hline Lantana camara $\mathrm{L}$ & Verbenaceae & Lantana & Shrub & $\begin{array}{l}\text { Plantation } \\
\text { areas }\end{array}$ \\
\hline Lawsonia inermis L. & Lythraceae & Mehedi & Shrub & Homestead \\
\hline Leea guineensis G.Don & Leeaceae & Phupharia & Shrub & $\begin{array}{l}\text { Plantation } \\
\text { areas }\end{array}$ \\
\hline $\begin{array}{l}\text { Leea macrophylla Roxb.ex } \\
\text { Hornem }\end{array}$ & Leeaceae & $\begin{array}{l}\text { Dhol } \\
\text { sumundro }\end{array}$ & Shrub & $\begin{array}{l}\text { Plantation } \\
\text { areas }\end{array}$ \\
\hline Leucas aspera (Willd.) Link & Lamiaceae & Dandakalash & Herb & Roadside \\
\hline Leucas lavandulaefolia Ress & Lamiaceae & Gaochia & Herb & Roadside \\
\hline Limnophila indica (L.) Druce & Scrophulariaceae & Pani karpur & Herb & \\
\hline Limonia acidissima $\mathrm{L}$ & Rutaceae & Koethbel & Tree & Homestead \\
\hline Lindernia antipoda (L.) Alston & Scrophulariaceae & Lindernia & Herb & $\begin{array}{l}\text { Cultivated } \\
\text { land }\end{array}$ \\
\hline Litsea glutinosa (Lour.) C.B.Rob & Lauraceae & Menda & Tree & $\begin{array}{l}\text { Plantation } \\
\text { areas }\end{array}$ \\
\hline $\begin{array}{l}\text { Ludwigia hyssopifolia (G. Don) } \\
\text { Excell }\end{array}$ & Onagraceae & Panilong & Herb & $\begin{array}{l}\text { Cultivated } \\
\text { land }\end{array}$ \\
\hline Ludwigia repens J.R.Forst. & Onagraceae & Mulsi & Herb & $\begin{array}{l}\text { Cultivated } \\
\text { land }\end{array}$ \\
\hline $\begin{array}{l}\text { Macrosolen cochinchinensis } \\
\text { (Lour.) Tiegh. }\end{array}$ & Loranthaceae & Porgassa & Herb & $\begin{array}{l}\text { Plantation } \\
\text { areas }\end{array}$ \\
\hline
\end{tabular}




\begin{tabular}{|c|c|c|c|c|}
\hline Scientific name & Family & Local name & Habit & Habitat \\
\hline Mangifera indica $\mathrm{L}$. & Anacardiceae & Aam & Tree & Homestead \\
\hline Melastoma malabathricum $\mathrm{L}$. & Melastomataceae & Futki & Shrub & $\begin{array}{l}\text { Plantation } \\
\text { areas }\end{array}$ \\
\hline Melia azedarach $\mathrm{L}$. & Meliaceae & Ghora neem & Tree & $\begin{array}{l}\text { Plantation } \\
\text { areas }\end{array}$ \\
\hline $\begin{array}{l}\text { Magnolia champaca (L.)Baill. ex } \\
\text { Pierre. }\end{array}$ & Magnoliaceae & Cahmpa & Tree & $\begin{array}{l}\text { Plantation } \\
\text { areas }\end{array}$ \\
\hline $\begin{array}{l}\text { Mikania cordata (Burm. f.) } \\
\text { B.L.Rob. }\end{array}$ & Asteraceae & Assamlata & Climber & $\begin{array}{l}\text { Plantation } \\
\text { areas }\end{array}$ \\
\hline Mimosa invisa Colla. & Mimosaceae & Bara lajjabati & Herb & $\begin{array}{l}\text { Plantation } \\
\text { areas }\end{array}$ \\
\hline Mimosa pudica $\mathrm{L}$. & Mimosaceae & Lajjabati & Herb & Roadside \\
\hline Mimusops elengi L. & Sapotaceae & Bokul & Tree & Homestead \\
\hline $\begin{array}{l}\text { Monochoria vaginalis (Burm. f.) } \\
\text { C. Presl }\end{array}$ & Pontederiaceae & Nukha & Herb & $\begin{array}{l}\text { Cultivated } \\
\text { land }\end{array}$ \\
\hline Moringa oleifera Lamk. & Moringaceae & Sajna & Tree & Homestead \\
\hline Mucuna pruriens (L.) DC. & Fabaceae & Alkushi & Climber & $\begin{array}{l}\text { Plantation } \\
\text { areas }\end{array}$ \\
\hline Murraya koenigii (L.) Spreng. & Rutaceae & Kamini & Shrub & $\begin{array}{l}\text { Plantation } \\
\text { areas }\end{array}$ \\
\hline Musa paradisiaca $\mathrm{L}$. & Musaceae & Kola & Herb & Homestead \\
\hline $\begin{array}{l}\text { Neolamarckia cadamba (Roxb.) } \\
\text { Bosser }\end{array}$ & Rubiaceae & Kadam & Tree & Homestead \\
\hline Nerium oleander L. & Apocynaceae & Korobi & Shrub & $\begin{array}{l}\text { Plantation } \\
\text { areas }\end{array}$ \\
\hline Nymphaea rubra Roxb. ex Andr. & Nymphaeaceae & Lal shaphla & Herb & $\begin{array}{l}\text { Plantation } \\
\text { areas }\end{array}$ \\
\hline Nypa fruticans Wurmb. & Arecaceae & Golpata & Shrub & $\begin{array}{l}\text { Mangrove } \\
\text { areas }\end{array}$ \\
\hline Ocimum terviflorum $\mathrm{L}$ & Lamiaceae & Tulsi & Herb & Homestead \\
\hline Opuntia dillenii (Ker Gawl.) Haw. & Cactaceae & Phanimansa & Herb & $\begin{array}{l}\text { Plantation } \\
\text { areas }\end{array}$ \\
\hline Oryza sativa $\mathrm{L}$. & Poaceae & Dhan & Herb & $\begin{array}{l}\text { Cultivated } \\
\text { land }\end{array}$ \\
\hline Oxalis corniculata $\mathrm{L}$. & Oxalidaceae & Amrul & Herb & $\begin{array}{l}\text { Cultivated } \\
\text { land }\end{array}$ \\
\hline Pandanus foetidus Roxb. & Pandanaceae & Keya kanta & Herb & $\begin{array}{l}\text { Mangrove } \\
\text { areas }\end{array}$ \\
\hline Panicum notatum Retz. & Poaceae & Panicum & Herb & $\begin{array}{l}\text { Cultivated } \\
\text { land }\end{array}$ \\
\hline Paspalum distichum $\mathrm{L}$. & Poaceae & Gitlaghas & Herb & $\begin{array}{l}\text { Cultivated } \\
\text { land }\end{array}$ \\
\hline Passiflora foetida L. & Passifloraceae & Jhumka lata & Climber & $\begin{array}{l}\text { Plantation } \\
\text { areas }\end{array}$ \\
\hline Polygonum pubescens Blume & Polygonaceae & Bish kata & Herb & $\begin{array}{l}\text { Plantation } \\
\text { areas }\end{array}$ \\
\hline $\begin{array}{l}\text { Polygonum hydropiper }(\mathrm{L} .) \\
\text { Delarbne }\end{array}$ & Polygonaceae & Lal-kukri & Herb & $\begin{array}{l}\text { Cultivated } \\
\text { land }\end{array}$ \\
\hline Phoenix sylvestris (L.) Roxb. & Arecaceae & Khejur & Tree & Homestead \\
\hline
\end{tabular}




\begin{tabular}{|c|c|c|c|c|}
\hline Scientific name & Family & Local name & Habit & Habitat \\
\hline $\begin{array}{l}\text { Phragmites karka (Retz.) Trin.ex } \\
\text { Steud }\end{array}$ & Poaceae & Nol & Herb & $\begin{array}{l}\text { Mangrove } \\
\text { areas }\end{array}$ \\
\hline Phyllanthus emblica L. & Euphorlsiaceae & Amlaki & Tree & Homestead \\
\hline Physalis minima $\mathrm{L}$. & Solanaceae & Fotka & Herb & $\begin{array}{l}\text { Plantation } \\
\text { areas }\end{array}$ \\
\hline Piper longum L. & Piperaceae & Pipul & Climber & Homestead \\
\hline Plumeria alba $\mathrm{L}$. & Apocynaceae & Katgolap & Tree & $\begin{array}{l}\text { Plantation } \\
\text { areas }\end{array}$ \\
\hline Pongamia pinnata (L.) Pierre & Caesalpiniaceae & Koroj & Tree & $\begin{array}{l}\text { Plantation } \\
\text { areas }\end{array}$ \\
\hline Pothos scandens L. & Araceae & Batilata & Climber & $\begin{array}{l}\text { Plantation } \\
\text { areas }\end{array}$ \\
\hline Premna esculenta Roxb. & Verbenaceae & Lallong & Shrub & $\begin{array}{l}\text { Plantation } \\
\text { areas }\end{array}$ \\
\hline Psidium guajava $\mathrm{L}$ & Myrtaceae & Piyara & Tree & Homestead \\
\hline $\begin{array}{l}\text { Pterospermum acerifolium } \\
\text { (L.)Willd. }\end{array}$ & Sterculiaceae & Muskunda & Tree & $\begin{array}{l}\text { Plantation } \\
\text { areas }\end{array}$ \\
\hline Rhizophora mucronata Lam. & Rhizophoraceae & Rhizophora & Tree & $\begin{array}{l}\text { Plantation } \\
\text { areas }\end{array}$ \\
\hline Rhynchostylis retusa (L.) Blume & Orchidaceae & Foxtail & Epiphyte & $\begin{array}{l}\text { Plantation } \\
\text { areas }\end{array}$ \\
\hline Ricinus communis L. & Euphorbiaceae & Reri & Herb & Homestead \\
\hline Saccharum spontaneum $\mathrm{L}$. & Poaceae & Kash & Herb & $\begin{array}{l}\text { Mangrove } \\
\text { areas }\end{array}$ \\
\hline Albizia saman (Jacq.) Merr. & Mimosaceae & Randi korai & Tree & $\begin{array}{l}\text { Plantation } \\
\text { areas }\end{array}$ \\
\hline Saraca thaipingensis Prain & Caesalpiniaceae & Ashok & Tree & $\begin{array}{l}\text { Plantation } \\
\text { areas }\end{array}$ \\
\hline $\begin{array}{l}\text { Schumannianthus dichotomus } \\
\text { (Roxb.) Gagnep. }\end{array}$ & Marantaceae & Patipata & Herb & Homestead \\
\hline $\begin{array}{l}\text { Scindapsus officinalis (Roxb.) } \\
\text { Schott }\end{array}$ & Araceae & Money plant & Herb & $\begin{array}{l}\text { Plantation } \\
\text { areas }\end{array}$ \\
\hline Scoparia dulcis L. & Scrophulariaceae & Bandhuni & Herb & Roadside \\
\hline Sesbania grandiflora (L.) Pers. & Fabaceae & Bakul ful & Shrub & Homestead \\
\hline Senna alata (L.) Roxb. & Caesalpiniaceae & Dadmordon & Shrub & Roadside \\
\hline Senna sophera (L.) Roxb. & Caesalpiniaceae & Kalkesunda & Herb & Roadside \\
\hline Senna tora $(\mathrm{L}$.$) Roxb.$ & Caesalpiniaceae & Chakunda & Herb & Roadside \\
\hline Perrisetum glaucum (L.) R. Br. & Poaceae & Bajra & Herb & $\begin{array}{l}\text { Cultivated } \\
\text { land }\end{array}$ \\
\hline Sida acuta Burm. f. & Malvaceae & Nakphul & Herb & $\begin{array}{l}\text { Cultivated } \\
\text { land }\end{array}$ \\
\hline $\begin{array}{l}\text { Sida cordata (Burm. f.) } \\
\text { Borss.Wallk. }\end{array}$ & Malvaceae & Junka & Herb & Roadside \\
\hline Smilax ovalifolia Roxb. ex D. Don. & Smilaceaae & Kumarilata & Climber & \\
\hline $\begin{array}{l}\text { Siplanthes acmella } \\
\text { (L.) Murray not (L.) L. }\end{array}$ & Asteraceae & Spilanthes & Herb & $\begin{array}{l}\text { Cultivated } \\
\text { land }\end{array}$ \\
\hline Solanum melongena $\mathrm{L}$. & Solanaceae & Begun & Herb & Homestead \\
\hline Solanum americanum Mill. & Solanaceae & Puti begun & Herb & $\begin{array}{l}\text { Plantation } \\
\text { areas }\end{array}$ \\
\hline
\end{tabular}




\begin{tabular}{|c|c|c|c|c|}
\hline Scientific name & Family & Local name & Habit & Habitat \\
\hline Solanum torvum $\mathrm{Sw}$. & Solanaceae & Gota begun & Shrub & $\begin{array}{l}\text { Plantation } \\
\text { areas }\end{array}$ \\
\hline Sonneratia apetala Buch.-Ham. & Sonneratiaceae & Keora & Tree & $\begin{array}{l}\text { Mangrove } \\
\text { areas }\end{array}$ \\
\hline Sonneratia caseolaris (L.) Engl. & Sonneratiaceae & Soila & Tree & $\begin{array}{l}\text { Mangrove } \\
\text { areas }\end{array}$ \\
\hline Spondias pinnata (L.f.) Kurz. & Anacardiaceae & Amra & Tree & Homestead \\
\hline Sterculia foetida $\mathrm{L}$. & Sterculiaceae & Jongli badam & Tree & $\begin{array}{l}\text { Plantation } \\
\text { areas }\end{array}$ \\
\hline Sterculia villosa Roxb. & Stercaliaceae & $\begin{array}{l}\text { Bsaket } \\
\text { badam }\end{array}$ & Tree & $\begin{array}{l}\text { Plantation } \\
\text { areas }\end{array}$ \\
\hline Steudnera colocasioides Hook. $\mathrm{f}$. & Araceae & Bishkachu & Herb & Homestead \\
\hline Streblus asper Lour. & Moraceae & Sheora & Tree & $\begin{array}{l}\text { Plantation } \\
\text { areas }\end{array}$ \\
\hline Swietenia mahagoni (L.) Jacq. & Meliaceae & Mehogoni & Tree & $\begin{array}{l}\text { Plantation } \\
\text { areas }\end{array}$ \\
\hline Syzygium cumini (L.) Skeels & Myrtaceae & Kalo Jam & Tree & Homestead \\
\hline $\begin{array}{l}\text { Tabernaemontana corymbosa } \\
\text { Roxb. ex Wall. }\end{array}$ & Apocynaceae & Tagar & Shrub & $\begin{array}{l}\text { Plantation } \\
\text { areas }\end{array}$ \\
\hline $\begin{array}{l}\text { Tabernaemontana divericata (L.) } \\
\text { R.Br.ex Roem \& Schult. }\end{array}$ & Apocynaceae & Tagar & Shrub & $\begin{array}{l}\text { Plantation } \\
\text { areas }\end{array}$ \\
\hline Tamarindus indica $\mathrm{L}$. & Caesalpiniaceae & Tetul & Tree & Homestead \\
\hline Tamarix gallica $\mathrm{L}$. & Amaricaceae & Nonajau & Shrub & $\begin{array}{l}\text { Mangrove } \\
\text { areas }\end{array}$ \\
\hline Tectona grandis L.f. & Verbenaceae & Segun & Tree & $\begin{array}{l}\text { Plantation } \\
\text { areas }\end{array}$ \\
\hline $\begin{array}{l}\text { Terminalia arjuna (Roxb. ex DC.) } \\
\text { Wight \& Arn }\end{array}$ & Combretaceae & Arjun & Tree & $\begin{array}{l}\text { Plantation } \\
\text { areas }\end{array}$ \\
\hline $\begin{array}{l}\text { Terminalia bellirica (Gaertn.) } \\
\text { Roxb. }\end{array}$ & Combretaceae & Bohera & Tree & $\begin{array}{l}\text { Plantation } \\
\text { areas }\end{array}$ \\
\hline Terminalia catappa $\mathrm{L}$. & Combretaceae & Kat badam & Tree & $\begin{array}{l}\text { Plantation } \\
\text { areas }\end{array}$ \\
\hline Terminalia chebula Retz. & Combretaceae & Haritoki & Tree & $\begin{array}{l}\text { Plantation } \\
\text { areas }\end{array}$ \\
\hline Thunbergia latifolia Roxb. & Acanthaceae & & Climber & $\begin{array}{l}\text { Plantation } \\
\text { areas }\end{array}$ \\
\hline $\begin{array}{l}\text { Thysanolaena maxima (Roxb. ex } \\
\text { Hornem.) Honda. }\end{array}$ & Poaceae & Phuljharu & Herb & $\begin{array}{l}\text { Cultivated } \\
\text { land }\end{array}$ \\
\hline Trewia nudiflora $\mathrm{L}$ & Euphorbiaceeae & Pitali & Tree & $\begin{array}{l}\text { Plantation } \\
\text { areas }\end{array}$ \\
\hline Triumfetta rhomboidea Jacq. & Tiliaceae & Banokra & Herb & $\begin{array}{l}\text { Plantation } \\
\text { areas }\end{array}$ \\
\hline Tridax procumbens $\mathrm{L}$. & Asteraceae & Tridhara & Herb & $\begin{array}{l}\text { Cultivated } \\
\text { land }\end{array}$ \\
\hline Typha elephantina Roxb. & Typhaceae & Hogla & Herb & $\begin{array}{l}\text { Cultivated } \\
\text { land }\end{array}$ \\
\hline Woodfordia fruticosa (L.) Kurz & Lythraceae & Dhatri-phul & Shrub & $\begin{array}{l}\text { Plantation } \\
\text { areas }\end{array}$ \\
\hline
\end{tabular}




\begin{tabular}{|c|c|c|c|c|}
\hline Scientific name & Family & Local name & Habit & Habitat \\
\hline Xanthium stromarium L. & Asteraceae & Ghagra & Herb & Roadside \\
\hline Urena lobata $\mathrm{L}$ & Malvaceae & Banokra & Herb & Roadside \\
\hline $\begin{array}{l}\text { Zingiber montanum (J. Koenig.) } \\
\text { Link ex A. Dietr. }\end{array}$ & Zingiberaceae & Paletara & Herb & $\begin{array}{l}\text { Plantation } \\
\text { areas }\end{array}$ \\
\hline Zingiber roseum (Roxb.) Rosc. & Zingiberaceae & Laltara & Herb & $\begin{array}{l}\text { Plantation } \\
\text { areas }\end{array}$ \\
\hline Ziziphus glabrata Heyne ex Roth & Rhamnaceae & Jangli kul & Shrub & $\begin{array}{l}\text { Plantation } \\
\text { areas }\end{array}$ \\
\hline Ziziphus jujuba Mill. & Rhamnaceae & Boroi & Tree & Homestead \\
\hline
\end{tabular}

such as Tamarix gallica,Pongamia pinnata, Barringtonia acutangula, Trewia nudiflora,Heritiera fomes, Nypa fruticans, Tamarindus indica, S. apetala, A. officinalis, S. caseolaris, Samanea saman, Albizia procera, Calophylum innophylum, Acacia nilotica, A. farnesiana, Casuarina equisetifolia and Rhizophora mucronata.

One embankment was made on the north site of the National Park to protect Kuakata municipal area from high tidal surges. Besides, many small roads and trails made by encroachers and forest department, and some plantation areas also established inside National Park. Embankment, plantation areas, small roads and trails were planted by the forest department using a number of both native and exotic species. The remarkable species are Samanea saman, Borassus flabelifer, Phoenix sylvestreis, Casuarina equisetifolia, Acacia nilotica A. farnesiana, A. auriculiformis, A. mangium, Eucalyptus camaldulensis, Albizia lebbeck, Artocarpus heterophyllus, Calophylum innophylum, Nerium indicum, Bauhinia purpurea, Delonix regia, Pongamia pinnata, Dalbergia sissoo, Ficus benghalensis, F. racemosa, Gmelina arborea, Terminalia arjuna, T. bellirica, T. chebula, Butea monosperma, Erythrina indica, Michelia champaca ,Swietenia mahagoni, Excoecaria agallocha, Cassia siamea and C. fistula. Some bushy plants were also found in this sides. The major species are Ricinus communis, Cajanus cajans, Cassia alata, Calotropis procera, Glycosmis pentaphylla, Clerodendrum viscosum, Datura metel, Hyptis suaveolens, Xanthium indicum, Solanum torvum, Ixora acuminate, Murraya koenigii and Ziziphus glabrata. Many climber species were also ornamented this sides. Most common species are Mikania cordata, Thunbergia fragrans, Pothos scandens, Hemidesmus indicus, Coccinia grandis, Dioscorea pentaphylla, Hodgsonia macrocarpa, Mucuna pruriens, Ficus pumila, Hedyotis scandens and Cuscuta reflexa.

In the Kuakata National Park, 383 encroachers have occupied of forest land and made homes. Each homestead was planted by a good number of tree species. The appearance of such homestead looks like a segment of mini forest. During our survey Moringa oleifera, Acacia nilotica, Aegle marmelose, Albizia lebbeck,Albizia procera, Samanea saman, Anacardium occidentale, Annona reticulate, Borassus flabellifer, Anthocephalus cadamba, Aphanamixis polystachya, Areca catechu, Artocarpus chaplasha, Artocarpus heterophyllus, Averrhoa carambola, Azadirachta indica, Bambusa tulda, Citrus maxima, 
Cocos nucifera, Elaeocarpus robustus, Ficus racemosa,Phoenix sylvestreis, Ziziphus mauritiana, Trewia nudiflora, Terminalia chebula, T. bellirica,Tamarindus indica, Syzygium cumini, Swietenia mahagoni, Spondias pinnata, Psidium guajava, Melia azederach, Mangifera indica, Lawsonia inermis, Erythrina indica and Diospyros malabarica were recorded.

Aside from plantation areas and homesteads, some areas are used as cultivated land. Local people and encroachers use such land ones in a year for rain fed aman rice cultivation. The most common plants recorded were Enhydra fluctuans, Eclipta alba, Centella asiatica, Blumea lacera, Tridax procumbe, Heliotropium indicum, Ludwigia repens, Oxalis corniculata, Echinochloa colonum, Oryza sativa, Panicum notatum, Setaria glauca and Bacopa monnieri and also a good number of sedges and grasses. In summer the land was sheltered by a number of herbaceous plants. Among them the common species were Xanthium indicum, Thysanolaena maxima, Ischaemum indicum, Echinochloa colonum, Sida acuta,Euphorbia hirta, Kyllinga nemoralis, Fimbristylis dichotoma, Cyperus cyperoides, Commelina benghalensis, Blumea membranacea and Paspalum distichum. A rare occurrence of Typha elephantina (Hogla) and Phragmitis karka (Nol) was also recorded in the wetland.

The four species namely Tamarix gallica, Calophylum inophylum, Typha elephantina and Phragmitis karka were found to be rare in the National Park. To authenticate their status further comprehensive survey is needed. A good number of medicinal plants were identified which plays important role for the primary healthcare of local people in and around the National Park. Priority should be given for their conservation. The recorded common species in the National Park were Adhatoda zeylanica, Andrographis paniculata, Achyranthes aspera, Phyllanthus emblica, Ocimum sanctum, Ricinus communis, Azadirachta indica, Aegle marmelos, Alstonia scholaris, Holarrhena antidysenterica, Sonneratia apetala, S. caseolaris, Nypa fruticans, Centella asiatica, Mangifera indica, Scoparia dulcis, Mikania cordata, Ipomoea fistolusa, Terminalia arjuna, T. chebula, T. belliricha, Cassia alata, Diilenia indica, Cynodon dactylon, Colocasia esculenta and Ficus racemosa.

Exotics and invasive species are a component of total floristic composition of the National Park. Some exotics, such as Acacia auriculiformis, A. mangium, Eucalyptus camaldulensis and Cassia siamea were planted in the National Park area. Invasive species of the National Park were found to be Eichhornia crassipes, Mikania cordata (Refugeelota), Ipomoea fistulosa, Ageratum conyzoides, Croton bonplandianum and Xanthium indicum. Such species are a challenge to the management of the plant diversity of the National Park. A good number of wildlife supporting plant species namely Sonneratia apetala, S. caseolaris, Avicenneia alba, Ficus benghalensis, F. racemosa, F. virens, Phoenix sylvestreis, Syzygium cummuni, Butea monosperma, Artocarpus chaplasha and Tamarindus indica was recorded from the National Park. Such species play an important role in conservation of biodiversity. 
Based on observations and discussion with local people and foresters it is evident that erosion is major threat to the National Park. The south and west sides of the National Park are facing high erosion due to regular sea wave actions. The species planted there are Acacia nilotica (Babla), A. farnesiana (Khaia Babla), A. auriculiformis (Akashmoni), Pongamia pinnata (Koroj), Barringtonia acutangula (Hizol) and Trewia nudiflora (Pitali) all of which are fresh water enduring species. Initially such species were doing better in producing branches and canopy. But their root systems are poorly developed. During high tide period the wave actions made them uprooted easily. Mangrove species like Sonneratia apetala (Keora), S. caseolaris (Soila), A. officinalis (Baen) and Ex. agallocha (Gewa) were found to grow well in the intertidal zone because they have strong root systems and can survive with high wave action during rainy season. Facilities and man power of local forest department are not much adequate. Introduction of exotics by forest department is also visible. Grazing by buffalos in the mangrove forest areas, plantation areas and newly accreted lands were also observed.

With the purpose of management of the National Park local knowledge based policy is very essential. During the field trips we discussed with local forest personals, local elites and general people to find some clues for formulating recommendations. The suggestions which are made based on our visit experiences are: to undertake short term and long term management plans, to install geo-tube or geo-bag on the south and west sites for protecting forest degradation and soil erosion, to develop eco-tourism, to ensure security for tourist, to provide visitor use for educational, cultural and recreational purposes at a level which will not cause significant biological or ecological degradation to the biodiversity, to create the sources of fresh water both for human and wildlife, to establish watch towers to enjoy sun rises, sun sets and natural views, to record local knowledge from the elders about nature and adaptation and to record health care knowledge of local people, to create awareness programs about environment, biodiversity and wildlife, to accelerate plantation programs using local species, to provide risk allowance for the people who involved in forest management process, to increase capacity of forest and forest personals, to detect and remove invasive species, to avoid exotics in plantation programs, to arrange traditional knowledge based cultural program, to create traditional medicinal knowledge sharing programs, to relocate encroachers from the park, finally to ensure land ownership and forest territory using GIS map.

The present work on the assessment of angiosperm diversity in Kuakata National Park is the first attempt. The record of total 265 angiosperm species in the park is very preliminary. We expected more angiosperm species yet to be identified. It is not possible to give a concrete conclusion based on such preliminary results. Long term floristic survey is necessary to cover all the component of the angiosperms and also other group of plants. 


\section{Acknowledgements}

Bangladesh Forest Department and local forest offices of Patuakhali Coastal Forest Division and Kuakata National Park are greatly acknowledged for financial support and facilities. The authors are also grateful to the Wildlife Center of Bangladesh Forest Department for implementing research work.

\section{References}

Ahmed, Z.U., Z.N.T. Begum, M. A. Hassan, M. Khondker, S. M.H. Kabir, M. Ahmad, A.T.A. Ahmed, A.K.A. Rahman and E.U. Haque (Eds). 2008a. Encyclopedia of Flora and Fauna of Bangladesh, Vol. 6. Angiosperms: Dicotyledons (Acanthaceae - Asteraceae). Asiatic Society of Bangladesh, Dhaka, pp. 1-408.

Ahmed, Z.U., M.A. Hassan, Z.N.T. Begum, M. Khondker, S.M.H. Kabir, M. Ahmad, A.T.A. Ahmed, A.K.A. Rahman and E.U. Haque (Eds). 2008b. Encyclopedia of Flora and Fauna of Bangladesh, Vol. 12. Angiosperms: Monocotyledons (Orchidaceae Zingiberaceae). Asiatic Society of Bangladesh, Dhaka, pp. 1-552.

Ahmed, Z.U., M.A. Hassan, Z.N.T. Begum, M. Khondker, S.M.H. Kabir, M. Ahmad, A.T.A. Ahmed, A.K.A. Rahman and E.U. Haque (Eds). 2009b. Encyclopedia of Flora and Fauna of Bangladesh, Vol.7. Angiosperms: Dicotyledons (Balsaminaceae Euphorbiaceae). Asiatic Society of Bangladesh, Dhaka, pp. 1-546.

Ahmed, Z.U., M.A. Hassan, Z.N.T. Begum, M. Khondker, S.M.H. Kabir, M. Ahmad, A.T.A. Ahmed, A.K.A. Rahman and E.U. Haque (Eds). 2009c. Encyclopedia of Flora and Fauna of Bangladesh, Vol.8. Angiosperms: Dicotyledons (Fabaceae -Lythraceae). Asiatic Society of Bangladesh, Dhaka, pp. 1-478.

Ahmed, Z.U., M.A. Hassan, Z.N.T. Begum, M. Khondker, S.M.H. Kabir, M. Ahmad, A.T.A. Ahmed, A.K.A. Rahman and E.U. Haque (Eds). 2009d. Encyclopedia of Flora and Fauna of Bangladesh,Vol. 9. Angiosperms: Dicotyledons (Magnoliaceae - Punicaceae). Asiatic Society of Bangladesh, Dhaka, pp. 1-488.

Ahmed, Z.U., M.A. Hassan, Z.N.T. Begum, M. Khondker, S.M.H. Kabir, M. Ahmad, A.T.A. Ahmed, A.K.A. Rahman and E.U. Haque (Eds).2009e. Encyclopedia of Flora and Fauna of Bangladesh, Vol. 10. Angiosperms: Dicotyledons (Ranunculaceae Zygophyllaceae). Asiatic Society of Bangladesh, Dhaka, pp. 1-580.

Ahmed, Z.U., M. Khondker, Z.N.T. Begum, M.A. Hassan, S.M.H. Kabir,M. Ahmad, A.T.A. Ahmed and A.K.A. Rahman (Eds). 2009a. Encyclopedia of Flora and Fauna of Bangladesh, Vol. 4. Algae: Charophyta-Rhodophyta (Achnanthaceae- Vaucheriaceae). Asiatic Society of Bangladesh, Dhaka, pp. 1-543.

Akter, A. and M.I. Zuberi. 2009. Invasive alien species in northern Bangladesh: Identification, inventory and impacts. International journal of biodiversity and conservation.1(5): 129134.

Alexiades, M.N. (Ed.).1996. Selected Guidelines for Ethno botanical Research: A Field Manual. The New York Botanical Garden, New York.

Ara, H., B. Khan and S.N. Uddin (Eds.). 2013. Red data book of vascular plants of Bangladesh, Vol.2. Bangladesh National Herbarium, Dhaka, Bangladesh. p.280.

Balick, M.J., A. B. Anderson and M. F.da Silva.1982. Plant taxonomy in Brazilian Amazonia: The state of systematic collection in regional herbaria. Brittonia 14: 463-477.

BFD, (Bangladesh Forest Department) 2006. Master plan of Kuakata Eco-Park. Bangladesh Forest Department, Agargaon, Dhaka, Bangladesh. pp.5-6.

BFD, (Bangladesh Forest Department) 2012. State of Bangladesh's forest protected areas. IPAC and Bangladesh Forest Department, Agargaon, Dhaka, Bangladesh. 63. 
Cronquist, A. 1981. An integrated system of classification of flowering plants. Columbia University Press, New York, p. 1262.

Goldsmith, F.B. and C.M. Harrison.1976.Description and analysis of vegetation .In: Champman SB (ed.). Methods in plant ecology. Blackwell, Oxford, pp. 85-155.

Hossain, M.K. and M.K. Pasha. 2004. An account of the exotic flora of Bangladesh. Journal of forestry and environment. 2: 99-115.

Hyland, B.P.M. 1972. A technique for collecting botanical specimens in rain forest. Flora Malesiana Bulletin. 26: 2038-2040.

Khan, M.S. and A. M.Huq. 2001. The vascular flora of Chunati Wildlife Sanctuary in south Chittagong, Bangladesh. Bangladesh J. Plant. Taxon. 8(1): 47-64.

Khan, M.S., M.M. Rahman and M.M. Ali (eds.). 2001. Red Data Book of Vascular Plants of Bangladesh. Bangladesh National Herbarium. 179.

Khan, M.S., M.M. Rahman, A.M. Huq, M.M.K Mia and M.A.Hassan.1994. Assessment of biodiversity of Teknaf game reserve in Bangladesh focusing on economically and ecologically important plants species. Bangladesh J. Plant.Taxon.1(1): 21-33.

Rahman, M.O. and M.A. Hassan.1995.Angiospermic flora of Bhawal Narional Park, Gazipur, Bangladesh. Bangladesh J. Plant Taxon. 2(1\&2): 47-79.

Sajib, N.H., S.B. Uddin and M.K. Pasha. 2015. Angiospermic Plant Diversity of Sandwip Island, Chittagong, Bangladesh. J. Asiat. Soc. Bangladesh, Sci. 41(2): 133-153.

Siddiqui, K.U., M.A.Islam, Z.U.Ahmed, Z.N.T Begum, M.A. Hassan, M. Khondker, M. M. Rahman, S.M.H Kabir, M. Ahmad, A.T.A Ahmed, A.K.A Rahman and E.U. Haque (Eds).2007. Encyclopedia of Flora and Fauna of Bangladesh. Vol.11. Angiosperms: Monocotyledons (Agavaceae -Najadaceae). Asiatic Society of Bangladesh, Dhaka, pp. 1399.

Uddin, M.Z., M. F.Alam, A.S.M. Rahman and M.A. Hassan. 2011. Plant Biodiversity of Fashiakhali Wildlife Santuary, Bangladesh. Accepted for publication in First Bangladesh Forestry Congress Proceeding 2011. pp.129-141.

Uddin, S.B. and M.A. Rahman.1999. Angiospermic flora of Himchari National Park, Cox's Bazar, Bangladesh. J. Plant Taxon. 6(1): 31-68.

Uddin, M.Z. and M.A. Hassan.2010. Angiosperm diversity of Lawachara National Park (Bangladesh): a preliminary assessment. Bangladesh J. Plant Taxon. 17 (1): 9-22.

Uddin, M.Z. and M.A. Hassan. 2004. Flora of Rema-Kalenga Wildlife Sanctuary. IUCN Bangladesh Country Office, Dhaka, Bangladesh, pp.1-120.

Uddin, M.Z., M.F. Alam, M.A. Rahman and M.A. Hassan. 2013. Diversity in angiosperm flora of Teknaf Wildlife Sanctuary, Bangladesh. Bangladesh J. Plant Taxon. 20(2): 145-162.

Uddin, S.N., M.S. Khan, M.A. Hassan and M.K. Alam.1998. An annotated checklist of angiospermic flora of Sitapahar at Kaptai in Bangladesh. Bangladesh J. Plant Taxon. 5(1): 13-46.

Uddin, M.Z., M.G. Kibria and M.A. Hassan. 2015. An assessment of angiosperm plant diversity of Nijhum Dweep (Island). J. Asiat. Soc. Bangladesh,Sci.41(1): 19-32.

Uddin, M.Z. and M. Abiabdullah. 2016. Taxonomic study on the angiosperms of Char Kukri Mukri Wildlife Sanctuary. J. Asiat. Soc. Bangladesh,Sci.42(2): 153-168. 